Psicologia Escolar

e Educacional
ARTIGO

DOI: http://dx.doi.org/10.1590/2175-35392021227267

Localizador - e227267

\title{
SUPORTE FAMILIAR COMO POSSÍVEL PREDITOR DAS ESTRATÉGIAS E DA MOTIVAÇÃO PARA APRENDER
}

\author{
Marcella das Neves Burgos ${ }^{1} \mathbb{D} ;$; Amanda Lays Monteiro Inácio ${ }^{2} \mathbb{D} ;$ Katya Luciane de Oliveira ${ }^{1} \mathbb{D} ;$ Makilim Nunes \\ Baptista ${ }^{2} \mathbb{D}$
}

\section{RESUMO}

O aluno com bom suporte familiar se sente mais vinculado à família e aos estudos, aumentando a probabilidade de apresentar maior motivação para aprender e tendência a ser mais estratégico no momento do aprendizado. Objetivouse investigar a correlação entre o suporte familiar, as estratégias de aprendizagem e a motivação para aprender e verificar se o suporte familiar prevê o uso dessas variáveis psicoeducacionais. Participaram 352 alunos matriculados no Ensino fundamental II de uma escola pública. Utilizou-se o Inventário de Percepção de Suporte Familiar - IPSF, Questionário Continuum Infantil e a Escala de Avaliação das Estratégias de Aprendizagem para o Ensino Fundamental - EAVAP-EF. Adotou-se o método de pesquisa descritivo, com delineamentos de levantamento e correlacional. Os resultados indicaram que os alunos que recebem suporte familiar tendem a fazer mais uso de estratégias de aprendizagem e apresentam mais motivação para aprender.

Palavras-chave: família; motivação; aprendizagem

\section{Support as a possible predictor of strategies and motivation to learn}

\begin{abstract}
Students with good family support feel more connected to their family and studies, increasing the probability of being more motivated to learn and tend to be more strategic at the time of learning. This study aimed to investigate the correlation among family support, learning strategies and motivation to learn and to verify whether family support includes the use of these psychoeducational variables. In this research, 352 students enrolled in Elementary School II of a public school participated. We used the Perception of Family Support Inventory - IPSF, Children's Continuum Questionnaire and the Assessment Scale of Learning Strategies for Elementary School - EVAP-EF. The descriptive research method was adopted, with survey and correlational designs. The results indicated that students who receive family support tend to make more use of learning strategies and they are more motivated to learn.
\end{abstract}

Keywords: family; motivation; learning

\section{Soporte familiar como posible predictor de las estrategias y de la motivación para aprender}

\section{RESUMEN}

El alumno con buen soporte familiar se siente más vinculado a la familia y a los estudios, aumentando la probabilidad de presentar más motivación para aprender y tendencia a ser más estratégico en el momento del aprendizaje. Se tuvo por objetivo investigar la correlación entre el soporte familiar, las estrategias de aprendizaje y la motivación para aprender y averiguar si el soporte familiar previene el uso de esas variables psico educacionales. Participaron 352 alumnos matriculados en la Enseñanza Básica II de una escuela pública. Se utilizó el Inventario de Percepción de Soporte Familiar - IPSF, Cuestionario Continuun Infantil y la Escala de Evaluación de las Estrategias de Aprendizaje para la Enseñanza Básica - EAVAP-EF. Se adoptó el método de investigación descriptivo, con delineamentos de recopilación y correlacional. Los resultados indicaron que los alumnos que reciben soporte familiar tienden a hacer más uso de estrategias de aprendizaje y presentan más motivación para aprender.

Palabras clave: familia; motivación; aprendizaje

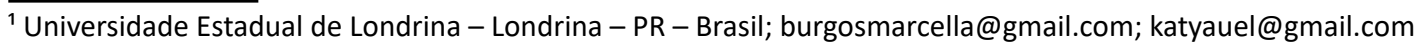

2 Universidade São Francisco - Campinas - SP - Brasil; amandalmonteiroo@gmail.com; makilim01@gmail.com
} 


\section{INTRODUÇÃO}

No âmbito educacional, atribui-se às famílias a capacidade de interferir positiva ou negativamente no desempenho escolar dos filhos. Porém, independente da estruturação familiar, a todas elas é conferido o papel de satisfazer as necessidades básicas e contribuir para o desenvolvimento social, a fim de que encontrem alicerce emocional e psíquico na construção de sua própria identidade. Muitos educadores acreditam que alunos que apresentam baixo desempenho escolar pertencem a famílias desestruturadas. Contudo, embora a família seja essencial ao longo do processo de escolarização, nem sempre aqueles que possuem histórico de fracasso são advindos de organizações familiares com esse perfil (Ribeiro, Ciasca, \& Capellato, 2016).

Somado a isso, na sociedade contemporânea são encontradas importantes mudanças na rotina familiar. $A$ título de exemplo, pode-se citar o fato de os pais trabaIharem o dia todo e, muitas vezes, não acompanharem de maneira contígua o processo de escolarização de seus filhos. Conforme mencionam Barros e Santos (2015), muitas famílias enfrentam dificuldades para acompanhar a trajetória escolar, o que pode vir a prejudicar o suporte familiar oferecido ao aluno.

Quando o aluno não recebe suporte da família para o estudo e apenas o encontra no ambiente escolar, pode apresentar desempenho inferior ou dificuldades no que tange ao desenvolvimento das habilidades socioemocionais e cognitivas, que são essenciais para se atingir a autorregulação da aprendizagem (Batista, Mantovani, \& Nascimento, 2015). Assim, a falta de suporte pode fazer com que o aluno encontre mais dificuldades em prestar atenção na sala de aula e para apresentar comportamentos sociais mais habilidosos. Por conta disso, é importante que a família ofereça estabilidade emocional e suporte ao estudo, a fim de que o aluno tenha capacidade para enfrentar os desafios pessoais e escolares de maneira satisfatória (Guidetti \& Martinelli, 2017).

No que se refere ao termo suporte familiar, para Baptista (2009) equivale à capacidade da família em oferecer proteção, comunicação, autonomia, respeito, interesse e segurança aos seus filhos, dentre outras características. Posto isto, o suporte familiar pode ser considerado como potencializador da educação, sendo capaz de afetar o desenvolvimento da criança, seu contexto de aprendizagem e, consequentemente, o seu rendimento escolar, tamanha sua relevância (Barros \& Santos, 2015; Roksa \& Kinsley, 2019).

Quando se busca encontrar relações entre o suporte familiar e as variáveis psicoeducacionais, é possível observar algumas associações exploratórias. Costa, Montiel e Bartholomeu (2016), por exemplo, encontraram relações entre suporte familiar (autonomia e adaptação familiar) e desempenho de leitura de crian- ças em alunos de 8 a 10 anos, sendo que a análise de regressão explicou quase $10 \%$ da variância $\left(r_{2}=0,098\right)$. As crianças auxiliadas pelos pais na tarefa de leitura são aquelas com melhor desempenho em leitura. Por sua vez, Ribeiro et al. (2016) encontraram associações entre recursos do ambiente familiar (passeio em família, livros e revistas, jogos pedagógicos, dentre outros) e desempenho escolar na escrita de alunos do $5^{\circ}$ ano do ensino fundamental $(r=0,345)$.

Diante do exposto pode-se afirmar que a vida escolar pode ser até certo ponto, influenciada pelo suporte familiar, e que o vínculo formado devido a esse suporte permite que $o$ aluno sinta-se, também, mais motivado a aprender. $\mathrm{O}$ aluno motivado consegue compreender os conteúdos de forma mais aprofundada e, para isso, faz uso de estratégias de aprendizagem que, quando utilizadas de forma adequada, facilitam a obtenção, retenção e o processamento da informação (Boruchovitch, 1999; Oliveira, Santos, \& Inácio, 2017).

No caso da motivação para aprender, esta se refere a um construto interno ou uma competência adquirida de acordo com a experiência do indivíduo na escola, sendo determinante para a qualidade da aprendizagem escolar (Brophy, 1987; Ryan \& Deci, 2017). Dentre as diversas teorias que versam sobre a temática, a Teoria da Autodeterminação (TAD) classifica a motivação nas vertentes intrínseca e extrínseca. Na primeira delas, os alunos tendem a empregar estratégias que exigem mais esforço e que lhes permitam processar a informação mais profundamente e, em geral, preferem tarefas desafiadoras. Enquanto isso, os alunos regulados pela motivação extrínseca exibem preferência por atividades com menor grau de dificuldade (Bzuneck, Oliveira, Rufini, \& Oliveira, 2015).

Para Maieski, Oliveira, Beluce e Rufini (2017), o estabelecimento de vínculos faz com que haja internalização de conceitos e regulações vindas de fora. Assim, internalizar é essencial para que haja a regulação de comportamento e a motivação seja otimizada no contexto escolar. Uma vez que o aluno se sente motivado e vinculado, esse sentimento está associado ao suporte familiar, podendo ser desencadeante de um fator motivacional mais presente nos estudos. Assim, levanta-se a hipótese de que por receberem suporte familiar, os alunos estabeleçam maiores vínculos com a aprendizagem. Esses vínculos permitem que se sintam mais motivados a aprender, utilizando-se de mais estratégias de aprendizagem no momento do estudo e consequentemente alcancem um melhor desempenho escolar (Castro, Miranda, \& Leal, 2016).

No que tange às estratégias de aprendizagem, podem ser concebidas como uma gama de atividades, comportamentos ou planejamento direcionados para o alcance de metas de aprendizagem pré-estabelecidas. Referem-se a uma atitude consciente e intencional 
advinda da tomada de decisões por parte do aprendiz, adaptada ao contexto e objetivos que se pretende alcançar, garantindo assim um bom desempenho escolar. Essas podem ser consideradas cognitivas, relacionando-se aos comportamentos de organização, armazenamento e elaboração de informações ou ainda metacognitivas, que são comportamentos relacionados à regulação, monitoração e planejamento dos próprios pensamentos durante o processo de aprender (Boruchovitch, 1999; Dembo, 1994).

O aluno deve diversificar as maneiras de estudar e recuperar os conteúdos, valendo-se de processos cognitivos. Assim sendo, é possível ainda, superar as dificuldades escolares para desenvolver atitudes de autoavaliação e melhora do desempenho escolar. Para tanto, faz-se necessário que o estudante conheça essas estratégias e saiba o momento certo em que cada uma delas deve ser utilizada (Boruchovitch, 1999; Oliveira et al., 2017).

Em face às conjecturas ora mencionadas, bem como à relevância do suporte familiar para a aprendizagem escolar, hipotetiza-se haver relação entre esse construto com as estratégias empregadas no momento do estudo e a motivação para aprender. Dessa forma, é possível que o aluno que se sente vinculado a sua família e recebe suporte familiar satisfatório, seja mais autônomo e motivado, alcançando, consequentemente, um melhor desempenho escolar. Nessa perspectiva, o objetivo do presente estudo foi investigar a correlação entre o suporte familiar com as estratégias de aprendizagem e com a motivação para aprender. Adicionalmente, pretendeu-se verificar se o suporte familiar é capaz de prever o uso dessas variáveis psicoeducacionais.

\section{MÉTODO}

\section{Participantes}

Participaram 355 alunos do 6 ao 9o anos do Ensino Fundamental II, com idades variando entre 10 e 16 anos $(M=12,6 ; D P=1,2)$. Desses, $39 \%(n=139)$ eram do sexo masculino e $60 \%(n=213)$ do sexo feminino, sendo que três participantes não responderam à questão. Os alunos pertenciam a uma escola pública do interior do Paraná e foram selecionados por conveniência. No que se refere ao ano escolar, o 6 o ano representou $25,6 \%$ ( $n$ = 91) da amostra, o 70 representou $34,6 \%(n=123)$, o 8 o ano retratou $25,1 \%(n=89)$ e o 90 ano $14,6 \%(n=52)$.

\section{Instrumentos}

Inventário de Percepção de Suporte Familiar (IPSF): Consiste em um instrumento de medida elaborado e validado para o Brasil por Baptista $(2005,2009)$. 0 inventário é composto por 42 afirmações relacionadas a situações familiares, em que o sujeito deve marcar a frequência com que cada uma delas acontece em sua família. Os itens são distribuídos em 3 subescalas, sen- do elas: Afetivo-Consistente, Adaptação e Autonomia.

Adota-se para a análise e classificação de resultados do inventário o desempenho considerado baixo, sendo de 0 a 21 pontos na subescala Afetivo-Consistente. Para a subescala de Adaptação a pontuação seria de 0 a 18 pontos. Varia de 0 a 9 pontos na subescala Autonomia e, ainda, de 0 a 53 pontos na pontuação total. No caso do Desempenho considerado médio-baixo, tem-se para Afetivo-Consistente de 22 a 28 pontos, para Adaptação de 19 a 21 pontos, para Autonomia de 10 a 12 pontos e na pontuação total de 54 a 63 pontos. $O$ resultado médio-alto seria de 29 a 33 pontos na subescala AfetivoConsistente, de 22 a 23 pontos na subescala Adaptação, de 13 a 14 pontos na subescala Autonomia e de 64 a 70 pontos na pontuação total. Por fim, a pontuação considerada alta seria de 34 a 42 pontos na subescala Afetivo-Consistente, de 24 a 26 pontos na subescala Adaptação, de 15 a 16 pontos na subescala Autonomia e 71 a 84 pontos na pontuação total.

Continuum Infantil: Elaborado por Rufini, Bzuneck e Oliveira (2011), o instrumento é constituído por 25 itens, baseados na Teoria da Autodeterminação (TAD), os quais dizem respeito aos motivos que levam o aluno a frequentar a escola. É composto por 5 subescalas, sendo elas: Desmotivação, Motivação Extrínseca por regulação externa, Motivação Extrínseca por Regulação Introjetada, Motivação Extrínseca por Regulação Identificada e Motivação Intrínseca. Nesse instrumento, a escala é apresentada a partir do desenho de figuras geométricas de tamanhos crescentes, seguidas dos números de 1 a 5 . 0 instrumento foi validado por meio da Análise Fatorial Exploratória, realizada pelos autores.

Escala de Avaliação das Estratégias de Aprendizagem para o Ensino Fundamental-(EAVAP-EF): Elaborada por Oliveira, Boruchovitch e Santos (2010) é composta por 31 itens, dispostos em uma escala do tipo Likert de três pontos ( 0 "nunca", 1 "às vezes", 2 "sempre"). Possui três subescalas, a saber: Ausência de Estratégias Metacognitivas Disfuncionais, Estratégias Cognitivas e Estratégias Metacognitivas, além da pontuação total. $O$ instrumento apresenta evidências de validade, além de avaliação da fidedignidade.

\section{Procedimento}

A pesquisa encontra-se respaldada na Resolução 466/2012 e em seus complementos do Conselho Nacional de Saúde e aprovada pelo Comitê de Ética com Seres Humanos de uma universidade do Norte do Paraná. Os instrumentos foram aplicados pela pesquisadora, após a assinatura do Termo de Assentimento Livre e Esclarecido - TALE pelos alunos e do Termo de Consentimento Livre e Esclarecido - TCLE pelos responsáveis. A coleta foi realizada de forma coletiva, em sala de aula, em dias e horários agendados previamente pela instituição, sendo os instrumentos respondidos em aproximadamente 
50 minutos. Para os alunos dos 60 anos, foram lidas as questões dos três instrumentos, uma a uma, a fim de facilitar a compreensão dos participantes.

\section{Análise de dados}

Foram realizadas estatísticas descritivas e inferenciais por meio do programa SPSS (Statistical Package for the Social Sciences) versão 22.0. Inicialmente foi realizada uma análise de frequência para verificar as médias, desvios-padrão, pontuação mínima e máxima obtida pelos participantes. Na sequência, realizou-se a correlação de Pearson entre os escores dos instrumentos, estimando-se a magnitude dessas correlações tendo por base os pressupostos de Cohen (1988). Por fim, foi verificada, por meio da Análise de Regressão Linear simples, a capacidade de predição da pontuação nos instrumentos aplicados.

\section{RESULTADOS}

De acordo com o objetivo inicial, na primeira parte dos resultados avaliou-se a habilidade dos alunos nos instrumentos empregados. Posteriormente, a soma dos itens foi dividida pelo número de itens, tornando possível a comparação. Desse modo, os resultados da análise descritiva podem ser visualizados na Tabela 1.

No que tange a percepção de suporte familiar, identificou-se na escala total que a média de acertos foi 54,2 , com um desvio padrão de 12,2 . Verificou-se que o mínimo de acertos foi 20 e o máximo 80 , sendo a variação de pontos possíveis entre 0 e 84 . Com isso, pode-se observar que os alunos obtiveram pontuação acima da média da metade da pontuação possível, ou seja, 42 pontos.

Foram avaliadas, também, as estratégias de aprendizagem empregadas, cuja pontuação total poderia variar de 0 a 62. Identificou-se a média total dos alunos, que foi de 31,1 , com desvio padrão de 9,1 . Observa-se que a pontuação mínima foi de 8 e a máxima de 58 pontos. Com isso, nota-se que os alunos obtiveram pontuação dentro da média esperada, que seria de 31 pontos, ou seja, metade dos acertos possíveis. Com relação à escala que avalia a motivação para aprender, a pontuação poderia variar entre 0 e 25 pontos em cada uma das subescalas, sendo a maior média encontrada em relação a subescala de Regulação Identificada.

Com o objetivo de investigar a correlação entre o suporte familiar e os demais construtos, a saber, estratégias de aprendizagem e motivação para aprender, realizou-se a prova de correlação de Pearson. A Tabela 2 apresenta os dados inerentes aos coeficientes de correlação entre a pontuação no Inventário de Percepção de Suporte Familiar com a Escala de Avaliação das Estratégias de Aprendizagem para o Ensino Fundamental e com o Continuum Infantil.

Foram encontradas correlações positivas e negativas da subescala Afetivo-Consistente com todas as subescalas de Estratégias de Aprendizagem e Motivação para Aprender, sendo que a magnitude variou de pequena a média (Cohen, 1988). O mesmo resultado foi encontrado com as subescalas de Adaptação e a escala Total de suporte familiar, todos com resultados significativos. No que tange à subescala Autonomia, os resultados não foram significativos somente com Ausência de Estratégias Metacognitivas Disfuncionais e com Desmotivação, ambos da escala de Estratégias de Aprendizagem.

Com o objetivo de verificar se o suporte familiar pode prever o uso das variáveis psicoeducacionais do presente estudo, realizou-se a Análise de Regressão Linear Simples. A Tabela 3 apresenta os dados inerentes à análise realizada entre o Inventário de Percepção de Suporte Familiar e a Escala de Avaliação das Estratégias de Aprendizagem que apresentaram correlação

Tabela 1 Estatísticas Descritivas dos Escores dos Instrumentos.

\begin{tabular}{lccccc}
\hline Instrumento & $\begin{array}{c}\text { Variação dos } \\
\text { pontos }\end{array}$ & Média & $\begin{array}{c}\text { Desvio } \\
\text { Padrão }\end{array}$ & $\begin{array}{c}\text { Pontuação } \\
\text { máxima }\end{array}$ & $\begin{array}{c}\text { Pontuação } \\
\text { mínima }\end{array}$ \\
\hline Afetivo-Consistente & $0-42$ & 26,2 & 7,5 & 42 & 6 \\
Adaptação & $0-26$ & 18,8 & 4,7 & 26 & 4 \\
Autonomia & $0-16$ & 8,9 & 2,9 & 16 & 0 \\
Total & $0-84$ & 54,2 & 12,2 & 80 & 20 \\
Aus. E. A. Meta Disf. & $0-26$ & 12,8 & 5,3 & 26 & 1,0 \\
E. A. Cognitivas & $0-22$ & 8,3 & 4,5 & 20 & 0 \\
E. A. Metacognitivas & $0-14$ & 9,9 & 2,2 & 14 & 3,0 \\
Total & $0-62$ & 31,1 & 9,1 & 58 & 8 \\
Desmotivação & $0-25$ & 11,8 & 4,8 & 25 & 5,0 \\
Mot. Reg.Externa & $0-25$ & 13,1 & 4,5 & 25 & 5,0 \\
Mot. Reg.Introjetada & $0-25$ & 14,6 & 5,3 & 25 & 5,0 \\
Mot. Reg.Identificada & $0-25$ & 21,5 & 3,9 & 25 & 5,0 \\
Mot. Intrínseca & $0-25$ & 17,3 & 5,0 & 25 & 5,0 \\
\hline
\end{tabular}


Tabela 2. Índices de Correlações ( $r$ ) e Níveis de Significância $(p)$ entre o IPSF com a EAVAP-EF e com o Continuum Infantil.

\begin{tabular}{|c|c|c|c|c|}
\hline & Afetivo-Consistente & Adaptação & Autonomia & Total \\
\hline Aus. E. A. Meta. Disf. & $0,348 * * *$ & $0,432 * * *$ & 0,225 & $0,395 * * *$ \\
\hline E. A. Metacognitivas & $0,165^{*}$ & $0,373 *$ & $0,141 *$ & $0,159 *$ \\
\hline E. A. Cognitivas & $0,384 * * *$ & $0,224 * *$ & $0,416^{* *}$ & $0,345^{* *}$ \\
\hline E. A. Total & $0,447 * * *$ & $0,393 * *$ & 0,109 & $0,460 * * *$ \\
\hline Desmotivação & $-0,379 * *$ & $-0,388 * * *$ & $-0,091$ & \\
\hline Mot. Reg, Externa & $-0,321 * *$ & $-0,303 * *$ & $0,367^{* *}$ & \\
\hline Mot. Reg. Introjetada & $-0,226 * *$ & $-0,279 * *$ & $-0,110^{*}$ & \\
\hline Mot. Reg. Identificada & $0,262 * *$ & $0,251^{* *}$ & $0,395 * *$ & \\
\hline Mot. Intrínseca & $0,351 * *$ & $0,294 * *$ & $0,113^{*}$ & \\
\hline
\end{tabular}

Nota: Nível de significância $*=0,050 ; * *=0,01 ; * * *=0,001$.

Tabela 3 . Regressão Linear para Suporte Familiar e Estratégias de Aprendizagem.

\begin{tabular}{|c|c|c|c|c|c|c|}
\hline Variável Independente & $\mathbf{R}$ & $\mathbf{R}^{2}$ ajustado & $\mathbf{F}$ & Betaz & $\mathbf{t}$ & Sig \\
\hline \multicolumn{7}{|c|}{ Suporte Familiar Total e Estratégias de Aprendizagem } \\
\hline Aus. E.A. Meta Disf. & 0,395 & 0,153 & $F(1,284)=52,401$ & 0,395 & 7,239 & 0,001 \\
\hline E. A. Cognitiva & 0,345 & 0,116 & $F(1,287)=38,538$ & 0,345 & 6,208 & 0,001 \\
\hline E. A. Total & 0,460 & 0,208 & $F(1,270)=72,275$ & 0,460 & 8,501 & 0,001 \\
\hline \multicolumn{7}{|c|}{ Subescala Afetivo-Consistente e Estratégias de Aprendizagem } \\
\hline Aus. E.A. Meta Disf. & 0,348 & 0,118 & $F(1,299)=40,942$ & 0,348 & 6,399 & 0,001 \\
\hline E. A. Cognitiva & 0,384 & 0,145 & $F(1,302)=52,136$ & 0,384 & 7,221 & 0,001 \\
\hline E. A. Total & 0,447 & 0,197 & $F(1,286)=71,139$ & 0,447 & 8,434 & 0,001 \\
\hline \multicolumn{7}{|c|}{ Subescala Adaptação e Estratégias de Aprendizagem } \\
\hline Aus. E.A. Meta Disf. & 0,432 & 0,184 & $F(1,313)=71,626$ & 0,432 & 8,463 & 0,001 \\
\hline E. A. Total & 0,393 & 0,152 & $F(1,297)=54,305$ & 0,393 & 7,369 & 0,001 \\
\hline
\end{tabular}

significativa.

De acordo com a Tabela 3, verificou-se que o suporte familiar total prevê em 15\% a Ausência de Estratégias de Aprendizagem Metacognitivas Disfuncionais, em $11 \%$ as Estratégias de Aprendizagem Cognitivas e em 20\% a pontuação Total de Estratégias de Aprendizagem. Esse resultado indica que essas subescalas do IPSF predizem o uso de estratégias de aprendizagem pelos alunos da amostra pesquisada, ou seja, que os alunos que recebem mais suporte familiar fazem menos uso de Estratégias Metacognitivas Disfuncionais e mais uso de Estratégias Cognitivas no momento da aprendizagem. A subescala Afetivo-Consistente pode prever em $11 \%$ a ausência de Estratégias de Aprendizagem Metacognitivas Disfuncionais, em $14 \%$ as Estratégias de Aprendizagem Cognitivas e em 19\% a pontuação Total de Estratégias de Aprendizagem. Assim, os estudantes que possuem mais manifestações de afeto entre os membros da fa- mília fazem menos uso de Estratégias de Aprendizagem Metacognitivas Disfuncionais e mais uso de Estratégias de Aprendizagem Cognitivas e de Estratégias de Aprendizagem, de modo geral.

A subescala Adaptação foi preditora em $18 \%$ da subescala Ausência de Estratégias de Aprendizagem Metacognitivas Disfuncionais e em 15\% das Estratégias de Aprendizagem Total. Diante disso, os alunos que se encontram mais adaptados em suas famílias fazem menos uso de Estratégias de Aprendizagem Metacognitivas Disfuncionais e mais uso de Estratégias de Aprendizagem de forma geral.

A partir da análise dos dados da regressão linear para o suporte familiar e o Continuum Infantil, a Tabela 4 foi estruturada. Nela, são apresentados os dados quanto ao Suporte Familiar Total, Subescalas Afetivo-Consistente e Adaptação.

A Tabela 4 apresenta o Suporte Familiar Total pre- 
Tabela 4. Regressão Linear para Suporte Familiar e o Continuum Infantil.

\begin{tabular}{|c|c|c|c|c|c|c|}
\hline Variável Independente & $\mathbf{R}$ & $R^{2}$ ajustado & $\mathbf{F}$ & Betaz & $\mathbf{t}$ & Sig \\
\hline \multicolumn{7}{|c|}{ Suporte Familiar Total e Motivação para Aprender } \\
\hline Desmotivação & 0,419 & 0,173 & $F(1,288)=61,270$ & $-0,419$ & $-7,828$ & 0,001 \\
\hline Mot. Reg. Externa & 0,322 & 0,101 & $F(1,287)=33,084$ & 0,322 & $-5,752$ & 0,001 \\
\hline Mot. Intrínseca & 0,360 & 0,127 & $F(1,284)=42,407$ & 0,360 & 6,512 & 0,001 \\
\hline \multicolumn{7}{|c|}{ Subescala Afetivo-Consistente e Motivação para Aprender } \\
\hline Desmotivação & 0,379 & 0,141 & $F(1,301)=50,462$ & $-0,379$ & $-7,104$ & 0,001 \\
\hline Mot. Reg. Externa & 0,321 & 0,100 & $F(1,302)=34,632$ & $-0,321$ & 5,885 & 0,001 \\
\hline Mot. Intrínseca & 0,351 & 0,120 & $F(1,299)=42,063$ & 0,351 & 6,486 & 0,001 \\
\hline \multicolumn{7}{|c|}{ Subescala Adaptação e Motivação para Aprender } \\
\hline Desmotivação & 0,382 & 0,148 & $F(1,321)=56,676$ & $-0,388$ & 7,528 & 0,001 \\
\hline
\end{tabular}

vendo em $17 \%$ a subescala Desmotivação, em $10 \%$ a Motivação por Regulação Externa e em 12\% a Motivação Intrínseca. Com base nisso, tem-se que o aluno que recebe suporte familiar de forma considerada por ele como satisfatória, se sente menos desmotivado a aprender, e ainda, impulsiona-se a aprender por consequências externas e também pelas próprias atividades em si.

A subescala Afetivo-Consistente pode prever em $14 \%$ a subescala Desmotivação, em $10 \%$ a Motivação por Regulação Externa e em 12\% a Motivação Intrínseca. Assim, o aluno que recebe e percebe $o$ afeto entre membros na família, apresenta menos desmotivação no aprendizado, mais motivação para aprender por consequências externas e também mais motivação pelo prazer que tem em aprender. A subescala Adaptação do suporte familiar pode prever em $14 \%$ a subescala Desmotivação, o que significa que o aluno que se percebe mais adaptado a sua família, apresenta menos desmotivação no aprendizado.

\section{DISCUSSÃO}

Os objetivos do presente estudo foram investigar a correlação entre o suporte familiar com as estratégias de aprendizagem e com a motivação para aprender e verificar se o suporte familiar prevê o uso dessas variáveis psicoeducacionais. Mediante os resultados, evidenciou-se que os alunos participantes tiveram boa percepção de suporte familiar, também houve relação entre o suporte familiar percebido, a motivação e o uso de estratégias, sendo verificado, também, certo grau de dependência entre os construtos. Os dados serão discutidos na sequência com base na literatura científica pertinente.

No que se refere aos resultados do IPSF, os alunos apresentaram a maior média de pontuação na subescala Adaptação. Assim, pode-se afirmar que participantes da amostra pesquisada apresentam comportamentos positivos em relação às suas famílias, tais como respei- to, diálogo, aceitação, entre outros. A segunda maior pontuação ocorreu na subescala Afetivo-Consistente, sendo que, possivelmente, os alunos percebam os integrantes de suas famílias como sendo afetivos e interessados por suas atividades. Essa subescala também se refere à clareza nas regras familiares, consistência de comportamentos, comunicação, interação, respeito, entre outros. A menor pontuação manifestada foi na Autonomia, contudo, com média acima da metade dos pontos possíveis, assim, é provável que haja, nessa amostra representativa, uma percepção de privacidade e liberdade entre os membros da família (Baptista, 2005, 2009).

Em relação à avaliação das estratégias de aprendizagem, a amostra apresentou a maior média de pontuação para a subescala de Estratégias Metacognitivas, indicando que os alunos conseguem pensar sobre o próprio pensamento, escolhem e selecionam ações no momento de aprender. Quanto à Ausência de Estratégias de Aprendizagem Metacognitivas disfuncionais, a média ficou um pouco abaixo da metade de acertos, evidenciando que, possivelmente, os estudantes ainda recorram, mesmo que pouco, a essas estratégias disfuncionais no momento do estudo (Boruchovitch, 1999; Dembo, 1994).

Já na subescala de Estratégias Cognitivas, que se refere à organização dos estudos, armazenar e elaborar informações, os alunos da amostra apresentaram baixa pontuação. Esse resultado pode ser visto como negativo, visto que tais estratégias possuem um papel importante para a aquisição dos conhecimentos e consolidação da aprendizagem no contexto escolar (Dembo, 1994; Oliveira et al., 2017).

No que tange ao Continuum Infantil, os alunos apresentaram maior pontuação na subescala Motivação Extrínseca por Regulação Identificada, que é caracterizada pela possibilidade de o indivíduo conseguir assumir 
para si o valor da tarefa, identificando-se com ela. Tal fato pode ser considerado positivo, visto que a subescala em questão é a mais próxima da motivação intrínseca (Bzuneck et al., 2015). A segunda subescala mais pontuada foi a de Motivação Intrínseca, o que permite afirmar que os alunos também são impulsionados a se comportarem pelo prazer proporcionado pelas atividades escolares. Na sequência, a Motivação Extrínseca por Regulação Introjetada manifestou estar acima da metade da pontuação possível, o que evidencia que os estudantes se apresentam, eventualmente, motivados por pressões internas, tais como sentimento de culpa, ansiedade ou exigências ligadas à autoestima (Brophy, 1987; Rufini et al., 2011).

Na subescala Motivação Extrínseca por Regulação Externa, o resultado também está acima da metade de acertos possíveis, o que pode ser considerado negativo, pois consiste em realizar uma atividade com a finalidade de conquistar benefícios externos, ou ainda, evitar consequências desagradáveis. Já a subescala Desmotivação também foi pontuada, ainda que um pouco abaixo da metade dos pontos possíveis. Esse resultado evidencia que, contexto escolar da amostra pesquisada, não há falta de intenção e de iniciativa, pelo contrário, os alunos demonstraram motivação para aprender (Bzuneck et al., 2015; Rufini et al., 2011; Ryan \& Deci, 2017).

Quanto às correlações entre as subescalas do IPSF com o Inventário de Estratégias de Aprendizagem e com o Continuum Infantil, as magnitudes dos resultados foram consideradas pequenas e médias (Cohen, 1988), sendo em sua maioria significativas. As subescalas Afetivo-Consistente, Adaptação e escala Total do IPSF obtiveram correlação com todas as subescalas da EAVAP-EF. Desta feita, pode-se inferir que o aluno que percebe haver afeto na família, comportamentos positivos e liberdade entre os membros, tende a utilizar estratégias de aprendizagem, evitar as estratégias de distração e manifesta mais comportamentos e pensamentos capazes de influenciar na aquisição e recuperação eficaz das informações (Dembo, 1994; Oliveira et al., 2017).

Quanto ao Continuum Infantil, as correlações também foram consideradas pequenas e médias entre todas as subescalas do IPSF com o questionário de motivação, não sendo significativa somente em relação à Autonomia com a subescala de Desmotivação. Além disso, ressalta-se que a Desmotivação, Motivação por Regulação Externa e por Regulação Introjetada se correlacionaram negativamente com a subescala Afetivo-Consistente e de Adaptação, indicando que quanto mais afetos e comportamentos positivos são percebidos pelos estudantes em relação à suas famílias, menos desmotivados e/ou regulados por motivações externas eles se apresentam (Ryan \& Deci, 2017).

Por conseguinte, os resultados evidenciaram correlações positivas e significativas entre a motivação
Identificada e motivação Intrínseca com as subescalas Afetivo-Consistente, Adaptação e Autonomia do IPSF. Os dados evidenciam que os estudantes que se identificam com as tarefas e sentem prazer em realizálas, percebem seus familiares com comportamentos e afetos positivos, bem como a existência de privacidade e liberdade entre os membros da família. Conforme mencionado por Maieski et al. (2017), tais fatores estão relacionados a um maior vínculo com a aprendizagem. Além disso, na faixa etária do Ensino Fundamental existe certa autonomia condicional, na qual à medida que o aluno se comporta de maneira positiva, em relação a sua aprendizagem, é contemplado pela família, ou seja, suas recompensas externas possivelmente estão ligadas a receber maior Autonomia, ou seja, privacidade, liberdade, entre outros. (Baptista, 2005, 2009).

As análises de regressão realizadas indicaram índices de predição relevantes. O Inventário de Percepção de Suporte Familiar demonstrou prever em aproximadamente $20 \%$ o emprego das estratégias de aprendizagem. Isso significa dizer que, até certa medida, os alunos que recebem suporte familiar tendem a fazer uso dessas estratégias no ambiente escolar, o que pode contribuir para uma aprendizagem mais efetiva e facilitada (Boruchovitch, 1999; Oliveira et al., 2017). Os demais dados de predição para as estratégias indicaram que a subescala Afetivo-Consistente também demonstrou prever, em certa medida, a Ausência de Estratégias Metacognitivas Disfuncionais, as Estratégias Cognitivas e as estratégias Total. Além disso, a subescala de Adaptação evidenciou índices de predição para a Ausência de Estratégias Metacognitivas Disfuncionais e para as estratégias Total. Ressalta-se que esses dados são inaugurais da literatura científica e, apesar de sua relevância, merecem ser vistos com cautela.

Já em relação ao IPSF e o Continuum Infantil, houve relação de predição entre a escala total do suporte familiar com as subescalas de Desmotivação, Motivação Externa e Motivação Intrínseca. Isso significa dizer que o suporte familiar pode predizer, em certo ponto, a desmotivação dos estudantes, bem como suas demandas externas. De modo semelhante, pôde predizer a realização da atividade pelo prazer em si mesmo (motivação intrínseca), indicando a importância da variável do suporte enquanto um indicador de forte influência na aprendizagem e consequentemente no sucesso escolar (Castro et al., 2016; Roksa \& Kinsley, 2019).

Ademais, a subescala Afetivo-Consistente do IPSF foi capaz de predizer, em certa medida, as subescalas de Desmotivação, Motivação por Regulação Externa e Motivação Intrínseca. Assim, os estudantes que se sentem acolhidos e amparados por seus familiares, tendem a apresentar menor desmotivação, realizam as tarefas por conta dos ganhos externos, mas também pelo prazer advindo dessas atividades. Por fim, a subescala 
Adaptação foi capaz de predizer a de Desmotivação, até certo ponto, evidenciando que os comportamentos positivos incididos da família, como respeito, aceitação, entre outros, estão relacionados com as características motivacionais dos estudantes da amostra pesquisada (Bzuneck, 2015; Ryan \& Deci, 2017).

Face às considerações trazidas, observa-se que existem desafios psicoeducacionais a serem percorridos, tais como a implementação de programas de intervenção junto aos alunos, a fim de que façam mais uso das estratégias de aprendizagem no momento do estudo, instrumentalização dos professores e ainda orientação de pais e responsáveis. Apesar das escolas enfrentarem problemas como as dificuldades de aprendizagem, seria possível a superação desses obstáculos caso as instituições de ensino instruíssem os alunos sobre a melhor forma de estudar, ensinando-os quais estratégias empregar e como utilizá-las no momento do aprendizado (Boruchovitch, 1999; Oliveira et al., 2017).

Há que se problematizar que, de acordo com a Base Nacional Comum Curricular - BNCC (2019), que consiste no documento mais atual e de caráter normativo que define o conjunto de aprendizagens essenciais que todos os alunos devem desenvolver ao longo das etapas e modalidades da Educação Básica, os objetivos gerais do ensino se referem à promoção das capacidades relativas aos aspectos cognitivo, afetivo, físico, ético, estético, de atuação e de inserção social, de forma a expressar a formação básica necessária para o exercício da cidadania.

Por outro lado, é recorrente a percepção de um distanciamento do Estado para com a instituição escolar, sendo que esse primeiro acaba por imputar muitas vezes aos pais um papel para o qual eles não estão profissionalmente capacitados. Nessa perspectiva, problematizar as concepções sobre tal relação se faz necessário, pois por um lado, muitas vezes há o afastamento da escola de suas funções, bem como em contrapartida, há ocasiões em que se subtraem dos pais as funções de natureza afetiva e social, que também são educativas, mas não escolarizadas. Por essa via, denota-se a complexidade da discussão Escola e Família, aspecto esse que merece ser mais bem investigado.

O presente estudo não se encontra livre de limitações. A esse respeito, ressalta-se que a amostra foi estabelecida por conveniência e existe a necessidade de novas pesquisas que objetivem investigar a questão do gênero dos participantes como fator interferente de possíveis diferenças nos resultados. Outra limitação pode ser o fato de os estudantes responderem a um inventário em sala de aula, o que pode intercorrer do efeito de desejabilidade social. Assim, espera-se que em pesquisas futuras seja realizada uma avaliação a partir da relação estudante família num planejamento que envolva entrevistas, a fim de se compreender de forma mais característica essa percepção de suporte.
Por fim, aponta-se como avanço a compreensão do suporte familiar, que é uma variável sócio-familiar, associar-se com variáveis da aprendizagem, como é o caso da motivação e estratégias de aprendizagem. Quando se demonstra a relação existente entre esses construtos, se estreita a importante discussão que fomenta a parceria entre os pais e as instituições de ensino, sendo perceptível a originalidade e contribuição dos dados ora apresentados, também pelo fato de, até o presente momento, não serem encontrados estudos que abordassem a temática com os objetivos e a etapa de escolarização ora retratada.

\section{REFERÊNCIAS}

Baptista, M. N. (2005). Desenvolvimento do Inventário de Percepção de Suporte Familiar (IPSF): estudos psicométricos preliminares. Psico-USF, 10(1), 11-19. https://dx.doi.org/10.1590/S1413-82712005000100003

Baptista, M. N. (2009). Inventário de percepção de suporte familiar (IPSF): estudo componencial. São Paulo: Vetor.

Barros, I. C. O.; Santos, J. (2015). O. A importância da parceria família-escola no processo de ensino aprendizagem. Revista brasileira de educação e saúde, 5(2), 39-45. Recuperado de https://www.gvaa.com.br/revista/index.php/REBES/ article/download/4111/3641

Batista, E. C.; Mantovani, L. K. S.; Nascimento, A. B. (2015). Percepção de suporte familiar de alunos com histórico de reprovação escolar. Debates em Educação, 7(13), 50-70. http://dx.doi.org/10.28998/2175-6600.2015v7n13p50

Boruchovitch, E. (1999). Estratégias de aprendizagem e desempenho escolar: considerações para a prática educacional. Psicologia: Reflexão e Crítica, 12(2), 361-376. https://dx.doi.org/10.1590/S0102-79721999000200008

Ministério da Educação. Base Nacional Comum Curricular (2019). Recuperado de http://basenacionalcomum.mec. gov.br/abase/

Brophy, J. (1987). Synthesis of Research on Strategies for Motivating Students to Learn. Educational leadership, 45(2). 40-48. Recuperado de http://www.ascd.org/ASCD/ pdf/journals/ed_lead/el_198710_brophy.pdf

Bzuneck, J. A.; Oliveira, M. F. C.; Rufini, S. E.; Oliveira, K. L. (2015). Estrutura fatorial de uma Escala de Motivação de Adolescentes para Leitura. Avaliação Psicológica, 14(3), 375-383. DOI: 10.15689/ap.2015.1403.09

Castro, J. X.; Miranda, G. J.; Leal, E. A. (2016). Estratégias de aprendizagem dos estudantes motivados. Advances in Scientific and Applied Accounting, 9(1), 80-97. Recuperado de https://asaa.anpcont.org.br/index.php/asaa/article/ view/258

Cohen, J. (1988). Statistical power analysis for the behavioral sciences. Hillsdale, NJ: Erlbaum.

Costa, K.; Montiel, J. M.; Bartholomeu, C. S. M. (2016). Percepção do suporte familiar e desempenho em leitura e escrita de crianças do ensino fundamental. Revista Psicopedagogia, 33(101), 154-163. Recuperado de http://pepsic.bvsalud.org/scielo.php?script=sci_ 
arttext\&pid=S0103-84862016000200005\&lng=pt\&tlng=pt.

Dembo, M. H. (1994). Applying educational psychology (5a Ed). New York: Longman Publishing Group.

Guidetti, A. A.; Martinelli, S. C. (2017). Percepções Infantis: Relações entre Motivação Escolar e Suporte Familiar. PsicoUSF, 22(3), 515-525. https://dx.doi.org/10.1590/141382712017220311

Maieski, S.; Oliveira, K. L.; Beluce, A. C.; Rufini, S. E. (2017). Motivação de alunos do ensino fundamental: estudo de duas realidades culturais. Psicologia Escolar e Educacional, 21(3), 601-608. https://dx.doi.org/10.1590/2175353920170213111129

Oliveira, K. L.; Boruchovitch, E.; Santos, A. A. A. (2010). Escala de avaliação das estratégias de aprendizagem para o ensino fundamental - EAVAP-EF. São Paulo: Casa do Psicólogo.

Oliveira, K. L.; Santos, A. A. A.; Inácio, A. L. M. (2017). Estratégias de aprendizagem no ensino médio brasileiro: análise exploratória dos resultados. Revista de Estudios e
Investigación en Psicología y Educación, Extra(01), 337-341. https://doi.org/10.17979/reipe.2017.0.01.3041

Ribeiro, R.; Ciasca, S. M.; Capelatto, I. V. (2016). Relação entre recursos familiares e desempenho escolar de alunos do 5 o ano do ensino fundamental de escola pública. Revista Psicopedagogia, 33(101), 164-174. Recuperado de http://pepsic.bvsalud.org/scielo.php?script=sci arttext\&pid=S0103-84862016000200006\&Ing=pt\&tIng=pt.

Roksa, J.; Kinsley, P. (2019). Correction to: The Role of Family Support in Facilitating Academic Success of Low-Income Students. Research in Higher Education, 60(437). https:// dx.doi.org/10.1007/s11162-018-9525-z

Rufini, S. E.; Bzuneck, J. A.; Oliveira, K. L. (2011). Estudo de validação de uma medida de avaliação da motivação para alunos do ensino fundamental. Psico-USF, 16(1), 1-9. https://dx.doi.org/10.1590/S1413-82712011000100002

Ryan, R. M.; Deci, E. L. (2017). Self-determination theory: Basic psychological needs in motivation, development, and wellness. NewYork: Guilford Publications.

Recebido: 09 de agosto de 2019

Aprovado: 29 de maio de 2021 